\title{
Conditions of Observation of Whistler Emissions in Space
}

\author{
C. Krafft, R. Lundin* and G. Matthieussent \\ Laboratoire de Physique des Gaz et des Plasmas, Bât. 212, Université Paris-Sud, CNRS, \\ 91405 Orsay cedex, France \\ *Institute of Terrestrial Magnetism, Ionosphere and Radiowave Propagation, Academy of Sciences, \\ Troitsk, Moscow Region, 142092, Russia
}

\begin{abstract}
The peculiarities of whistler wave fields spontaneously radiated by electron beams artificially injected into the Earth's ionosphere are discussed. The conditions of registration of the whistler wave packets by a remote on board VLF receiver have been analyzed; they depend on mutual disposition of the electron gun and the VLF receiver as well as on the pitch angle of the injected electrons. It is shown that the internal frequency width of the whistler signal, connected with the ray representation of a wave field, depends on the distance between the emitting region of the beam and the receiver and determines the lowest bandwidth of the VLF receiver in use. It is found that near the double pole singularity at the Gendrin's velocity the energy loss of a single electron increases by a factor of the order of ratio of the background electron plasma frequency to the electron gyrofrequency. The estimations for the coherence length of the beam and the ratio of coherent to incoherent level of radiated whistler amplitude are also presented. The theoretical analysis of the whistler wave field near the beam front has revealed crucial differences between the registration pattern of high-frequency and whistler frequency emissions in active experiments similar to ARAKS [1] and APEX [2-3].
\end{abstract}

\section{INTRODUCTION}

A theoretical study of the conditions of observation of a whistler signal produced by an electron beam artificially injected in the ionospheric plasma was performed in order to apply the results to active space experiments and in particular to the Active Plasma EXperiment (APEX, [2-3]). The active phase of APEX was performed by russian physicists during 1992-1993 and is based on operation of two satellites: the main satellite contains the electron gun which injects the electron beam; the subsatellite receives the whistler signals at position $(R, Z)$, with distances $R$ and $Z$ to the main satellite respectively accross and along the magnetic field.

We consider the beam as an antenna current and discuss the problems of emission, propagation and registration by a remote receiver of highly dispersive electron whistler mode waves in an overdense ionospheric plasma. Thus the efficiency of radiation, the conditions governing the frequency bands available for the wave registration and the requirements for the internal frequency width of the narrow frequency filters on board the remote receiver are analyzed. The problem of determination of the appropriate dynamical range for the receiver in use is studied through the calculation of the ratio of coherent to incoherent amplitudes of the beam emission. The obtained results are useful for an effective planning of future space active experiments with injection of energetic electron beams into space plasmas.

\section{THE RADIATED WAVE FIELD NEAR THE REMOTE RECEIVER}

Refering to [4] for the detailed description of the calculation of the wave field radiated by a single test charged particle spiralling in a magnetized homogeneous plasma, the field corresponding to the semiinfinite electron beam emission can be obtained through the integration of a single particle field over the injection time $t_{0} t_{0}$ with a weighting function $I\left(t_{0}\right) / e$, where the $I\left(t_{0}\right)$ is the injection current and $e$ the electron charge. Final expressions for the electric and magnetic field spectral components of the beam emission at the receiver position $(R, Z)$ can be written as 


$$
\begin{gathered}
\left(\begin{array}{c}
\vec{E} \\
\vec{B}
\end{array}\right)=\frac{I_{0}}{e} \sum_{m=-\infty}^{\infty} \sum_{j=1}^{2} \int_{-\infty}^{\infty} d \omega \exp [i \tilde{F}(\omega, u)] \frac{G_{m}(\omega)}{k_{\perp 1}^{2}(\omega)-k_{\perp 2}^{2}(\omega)}\left(\begin{array}{l}
\overrightarrow{\mathrm{E}} \\
\overrightarrow{\mathrm{B}}
\end{array}\right) \\
\tilde{G}_{m}(\omega)=G_{m}(\omega) \exp \left[i(-1)^{j+1} k_{\perp j}(\omega) R\right] \\
\tilde{F}(\omega, u)=-\omega(t-Z / u)+(-1)^{j+1} k_{\perp j}(\omega) R+m Z \omega_{c} / u \\
\overrightarrow{\mathrm{E}}=\left(\begin{array}{c}
\frac{m}{U} H_{m}^{(j)}(U)+\delta_{x} H_{m}^{\prime(j)}(U) \\
-i \delta_{x} \frac{m}{U} H_{m}^{(j)}(U)-i H_{m}^{\prime(j)}(U) \\
i \delta_{z} H_{m}^{(j)}(U)
\end{array}\right), \quad \overrightarrow{\mathrm{B}}=\left(\begin{array}{c}
-\delta_{0} k \frac{m}{U} H_{m}^{(j)}(U)-k_{z} H_{m}^{\prime(j)}(U) \\
i k_{z} \frac{m}{U} H_{m}^{(j)}(U)+i k \delta_{0} H_{m}^{\prime(j)}(U) \\
i k_{\perp} H_{m}^{(j)}(U)
\end{array}\right)
\end{gathered}
$$

where components of vectors are expressed in cylindrical coordinates. The wave number is $\vec{k}\left(k_{1}, k_{z}\right)$; $H_{m}^{(j)}(U)$ and $H_{m}^{\prime(j)}(U)$ are the Hankel function of kind $j$ and of order $m$ and its derivative, respectively; their argument is $U=k_{\perp j}(\omega) R ; j=1,2$ numerates the two branches corresponding to the solutions of the whistler dispersion relation for $k_{\perp j}(\omega)$ at a fixed frequency $\omega$ and resonance condition $k_{z} u=\omega+m \omega_{c}(u$ is the parallel beam velocity and $\omega_{c}$ the electron gyrofrequency). The expressions for the function $\tilde{G}_{m}(\omega)$ and the polarization coefficients $\delta_{x}, \delta_{z}$ and $\delta_{0}$ are presented in [4].

The last integration over the frequency $\omega$ in (1) can be performed; for the case of wave recording by a remote receiver, by using the Stationary Phase Point (SPP) method. In this "far field" approximation $(U>>1)$, the main contribution to the integral (1) corresponds to the integration in the vicinity of the stationary phase points $\omega_{s}$, where the condition $\partial \tilde{F}(\omega, u) / \partial \omega=0$ is fulfilled. Then the calculated amplitudes of the radiated wave field will correspond to the amplitudes of a signal propagating with group velocity $\vec{v}_{G}\left(v_{G Z}, v_{G L}\right)$ from the beam to the receiver. The internal frequency width $\delta \omega_{s}$ of this signal, that is the size of the essential vicinity of the SPP frequency, can be estimated through the second derivative of the phase over the frequency as

$$
\delta \omega_{s}=\sqrt{\frac{2}{\left|\tilde{F}^{\prime \prime}(\omega, u)\right|}}
$$

This width is not infinitesimally small in the whistler frequency band: for the ARAKS experiment it reached a value of $40 \mathrm{kHz}$ [4]. To record the arriving whistler signal without distortion, the frequency filters of the remote receiver should embrace its main spectral components. Thus the condition $\delta \omega_{R} \geq \delta \omega_{S}$ should be satisfied ( $\delta \omega_{R}$ is the frequency width of the receiver's filter).

The amplitude of the signal corresponding to the frequency width $\delta \omega_{S}$ is collected mainly from the beam region of length

$$
L_{C}=u \sqrt{2\left|\tilde{F}^{\prime \prime}(\omega, u)\right|}
$$

The peculiarities of motion of the beam electrons inside this region can crucially influence on the level of the emitted signal. To receive high level signals one should provide a coherence in the motion of the beam electrons inside the considered region. Thus its length naturally defines the coherence length of the beam.

In the approximation of the SPP method one can find the emitting region of the beam which can be linked by the group velocity vector with the position of the receiver. In the case where the beam is injected into one hemisphere $(z>0)$, this circumstance allows to determine the frequency band limitations for the registered signal. They depend on the mutual disposition of the electron gun and the remote receiver. For an injection in the direction of the remote receiver, both types of resonances ( $m \geq 0$, and $m<0$ when the 
beam front has passed the receiver position) can produce an observable signal. For a backward injection only the emissions at $m<0$ can be recorded. The comparison between the calculated and the measured frequency bands can in principle allow to distinguish between different possible sources of whistler emissions during the injection cycle.

As it was shown in [4], the analysis of the recorded amplitude of the whistler waves as a function of the injection pitch angle $\theta_{P}$ can give a possibility to estimate the effective collision frequency $v$ of the background plasma electrons. The maximum value of the signal emitted at Cherenkov resonance (it is the only essential resonance for the case of an azimuthally homogeneous electron beam) corresponds to the parallel beam velocity

$$
u_{M A X}=2 R v /\left(\frac{\omega}{\omega_{c}} \sqrt{1-\frac{\omega^{2}}{\omega_{c}^{2}}}\right)
$$

At larger injection pitch angles the amplitude of the radiated electric field diminishes exponentionally. Then through an effective collision frequency $v$, the level of the turbulence excited in the background plasma during the injection can be estimated.

The power emitted by the beam as a function of its parallel velocity can be analyzed using (1). The power should increase when two roots $k_{11}$ and $k_{\perp 2}$ are close one to another [4-6]. This occurs in overdense plasmas when the parallel velocity of the beam electrons is equal to the Gendrin's velocity $v_{G E}=c \omega_{\varepsilon} / 2 \omega_{p}$ [5] (double pole case); $\omega_{p}$ is the electron plasma frequency. At this value, a large set of whistler waves can interact resonantly at Cherenkov resonance with those electrons which then loose their energy instantly. To resolve this infinity problem, one should notice that the possibility to be in resonance with an infinite group of whistler waves occurs only if one neglects the higher-order terms in the expansion of the whistler refractive index over the small parameter $\omega_{c} / \omega_{p}<<1$ in the so-called quasilongitudinal approximation for a dense plasma. Keeping these terms one can find that

$$
k_{12}^{2}-k_{\perp 1}^{2} \propto \sqrt{1-4 \frac{\omega_{p}^{2} u^{2}}{\omega_{c}^{2} c^{2}}\left(1+\frac{\omega_{c}^{2}}{2 \omega_{p}^{2}}-\frac{\omega^{2}}{\omega_{p}^{2}}\right)}
$$

So the integration over frequency in (1) near the double pole can be performed without any problems. The increase of energy loss near the Gendrin's velocity can be estimated approximately by a factor $\omega_{p} / \omega_{c}$.

Another interesting feature of the whistler radiated field, connected with whistler wave dispersion in an overdense plasma, is that near the leading beam front there is a Mach-cone structure for the wave field emitted at Doppler-shifted resonances; thus a sharp increase of the field can be registered during the beam front pass (for high frequency Langmuir waves this phenomenon was observed in the ARAKS experiment [1]). However, there is no Mach-cone structure for the whistler wave field emitted at Cherenkov resonance: this circumstance could correspond to the ARAKS experimental result where no any peak of amplitude in the whistler frequency band was registered.

Let us present the ratio $\mathrm{P}_{R}$ of the coherent to the incoherent amplitudes of the emitted field

$$
\mathrm{P}_{R}=\sqrt{\frac{I_{0}}{e}} \frac{\delta \omega_{S}}{2 \omega} \frac{1}{\sqrt{\delta \omega_{R}}}
$$

This value can be directly applied to the experimental measurements if it is possible to extract from the recorded signal the contribution of a single plasma mode at a single resonance $m$ and branch $j$. Here $\delta \omega_{R}$ is the receiver half bandwidth. For an optimized receiver (i.e. when $\delta \omega_{R}=\delta \omega_{S}$ ), $\mathrm{P}_{R}$ is some universal function of the experimental parameters. In the case of Cherenkov resonance, the expression for $\delta \omega_{S}$ can be simplified as

$$
\delta \omega_{s}=\omega_{c} \sqrt{\frac{2 u}{R \omega_{c} a_{j}}}\left(1-a_{j}^{2} \frac{\omega^{2}}{\omega_{c}^{2}}\right)^{3 / 4}, a_{j}=\frac{2}{P}\left(1-(-1)^{j} \sqrt{1-P}\right), P=4 \frac{\omega_{p}^{2}}{\omega_{c}^{2}} \frac{u^{2}}{c^{2}}
$$


For application to experiments, one can use the formula

$$
\mathrm{P}_{R}=1.77 \times 10^{7} \frac{\delta \omega_{S}}{\omega} \sqrt{\frac{5 I_{0}(A)}{\delta F_{R}(k H z)}}
$$

where $\delta F_{R}$ is the receiver half bandwidth in kilohertz and $I_{0}$ is the effective beam current in ampere.

Let us give some numerical estimations in application to the following APEX conditions: $E_{B}=10 \mathrm{keV}$ (beam energy), $\theta_{P}=85^{\circ}, I_{0}=0.1 \mathrm{~A}, R=10 \mathrm{~km}$ and $Z=8 \mathrm{~km}, \omega_{c} / 2 \pi=1145 \mathrm{kHz}, \omega_{p} / \omega_{c}=4$, $\omega / \omega_{c}=0.9$ (signal frequency), $m=0$ (Cherenkov resonance), $j=2$ (quasi-electrostatic whistler branch). The domain of observable frequencies corresponds to [893,1145] $\mathrm{kHz}$. Other values are: $\delta \omega_{S} / 2 \pi=\delta \omega_{R} / 2 \pi=20 \mathrm{kHz}, L_{C}=2 \mathrm{~km}, \mathrm{P}_{R}=4 \cdot 10^{4}$.

The case of a thin semi-infinite modulated electron beam is considered in [6-7].

\section{CONCLUSION}

We have found the conditions for an undistorted recording of the electromagnetic signals radiated during the active space experiments in the Earth ionosphere. The results are closely connected with the ray representation of the whistler wave field emitted by energetic electron beams, namely with the intrinsic frequency width of a signal propagating with group velocity.

The interpretation of the experimental records will be complicated due to the different possible channels of radiation of the whistler signals at the same frequency. In order to reduce to the minimum (or even to one) the possible channels of radiation compatible with the receiver in use, one should take care in planning the mutual disposition of the electron gun and the remote receiver, as well as the beam injection pitch angle and the registrated frequency bands .

\section{Acknowledgments}

One of the authors, B.L., acknowledges the finacial support from DRET and CNRS during his visit to the Laboratoire de Physique des Gaz et des Plasmas of the University Paris South, Orsay, France. The research described in this publication was made possible in part by Grant $N^{\circ}$ MK6000 from the International Science Foundation.

\section{References}

[1] Lavergnat J., The French-Soviet Experiment ARAKS: Main Results, Artificial Particle Beams in Space Plasma Studies (Plenum, New York, 1982) pp.87-94.

[2] Oraevsky V. et al,, J. Atmos. Terr. Phys. 5(3) (1994) 423-431.

[3] Dokukin V., The Scientific Instrumentation of the APEX Project, Proc of the International Conference on Active Experiment (Nauka, Moscow, 1992) pp.18-23.

[4] Lundin B. et al., J. Geophys. Res. 99 (1994) 14987-15003.

[5] Etcheto J. and Gendrin R., Planet Space Sci. 18 (1970) 777-784.

[6] Lundin B., Kraff C. and Matthieussent G., J. Geophys. Res. 100 (1995) 3703-3713.

[7] Krafft C., Lundin B., Matthieussent G. and Volokitin A., J. Phys. IV France, see this issue. 